\title{
Use of ordered mesoporous materials as tools for organic and bioorganic synthesis
}

\author{
Paul Handa, ${ }^{\text {a Tomasz Witula, }}$ Pedro Reis, ${ }^{\text {a,b }}$ and Krister Holmberg*a \\ ${ }^{a}$ Chalmers University of Technology, Department of Chemical and Biological Engineering, SE-412 \\ 96 Göteborg, Sweden \\ ${ }^{b}$ Nestlé Research Center, CH-1000, Lausanne 26, Switzerland \\ E-mail: kh@chalmers.se
}

Dedicated to Prof. Torbjörn Norin on his $75^{\text {th }}$ anniversary

\begin{abstract}
Ordered mesoporous materials are materials with well defined pore sizes in the range of 2 to $50 \mathrm{~nm}$. This paper shows that suspensions of particles of mesoporous materials can be useful tools for organic and bioorganic synthesis. They can be used to overcome reactant incompatibility, as is demonstrated for a reaction between 4-tert-butylbenzyl bromide and KI. The pores are impregnated with an aqueous solution of KI and the lipophilic reactant is present in the continuous apolar phase. The reaction occurs at the pore openings. The pores of the mesoporous material can also be used as host for a homogeneous catalyst. The catalyst-loaded particles are kept as a suspension in a medium that contains the reactants. This principle is demonstrated for a Heck-type reaction with a watersoluble rhodium catalyst inserted into the pores of mesoporous silica. It is also shown to work well for a 1,4-conjugate addition between two water-soluble reactants, 4-carboxyphenylboronic acid and methyl acrylate, in which case a toluene-soluble rhodium catalyst was entrapped in the pores of hydrophobized mesoporous silica. The same principle was also used for biocatalysis. Lipase was inserted into either hydrophilic or hydrophobic pores and used for both ester synthesis and ester hydrolysis. Finally, palladium nanoparticles were deposited inside the pores of mesoporous carbon and used as catalyst for a Sonogashira reaction.
\end{abstract}

Keywords: Ordered mesoporous materials, homogeneous catalysis, heterogeneous catalysis, biocatalysis, Heck reaction, Sonogashira reaction

\section{Introduction}

Reactions forming new carbon-carbon bonds are central in synthetic organic chemistry. Many such reactions involve a homogeneous catalyst, usually a metal-organic compound, while some are based on heterogeneous catalysis with the catalytically active sites present on a support material. In some cases, as for the Sonogashira reaction, it is not absolutely clear if the reaction is catalyzed by deposited active material, in this case metallic palladium (heterogeneous catalysis), or whether the 
solid catalyst is just a reservoir for the true catalyst, which continuously leaches out into the reaction solution (homogeneous catalysis).

We have been interested in using ordered mesoporous materials as hosts for catalysts that promote the formation of carbon-carbon bonds. Mesoporous materials are materials with well defined pore sizes in the 2 to $50 \mathrm{~nm}$ regime. ${ }^{1}$ The material can have hexagonal or cubic geometry and has a high specific surface area, typically in the range of 500 to $1000 \mathrm{~m}^{2} / \mathrm{g}$.

In this communication, which is an account of recent and ongoing work, we first demonstrate that different types of mesoporous material, in the form of a suspension, can be used to overcome incompatibility problems in organic synthesis, demonstrated by a typical $\mathrm{S}_{\mathrm{N}} 2$ reaction. We then proceed to three types of carbon-carbon coupling reactions: a Heck type reaction catalyzed by a water-soluble rhodium complex inserted into the water-filled pores of the material, an 1,4-conjugate addition using an apolar rhodium catalyst inserted in the pores of hydrophobized mesoporous silica, and a Sonogashira reaction with palladium in the form of nanoparticles deposited inside the pores of mesoporous carbon. Finally, we report results from a work where an enzyme was heterogenized into the pores of mesoporous silica or alumina, which were either hydrophilic or hydrophobic. In all reactions involving catalyst-loaded particles these were present in the form of a concentrated slurry and a solvent of opposite polarity, which contained the reactants, constituted the continuous phase. The reactions occurred at the pore openings.

\section{Results and Discussion}

\section{Use of ordered mesoporous oxide materials as a way to overcome reactant incompatibility}

The reaction between 4-tert-butylbenzyl bromide (4-TBBB) and potassium iodide (KI) was performed in a concentrated slurry of finely divided mesoporous oxide material in an apolar medium, see Figure 1. The pores were impregnated with an aqueous solution of KI and the lipophilic reactant, 4-TBBB, was present in the continuous phase. A detailed experimental procedure can be found in Ref. 2.

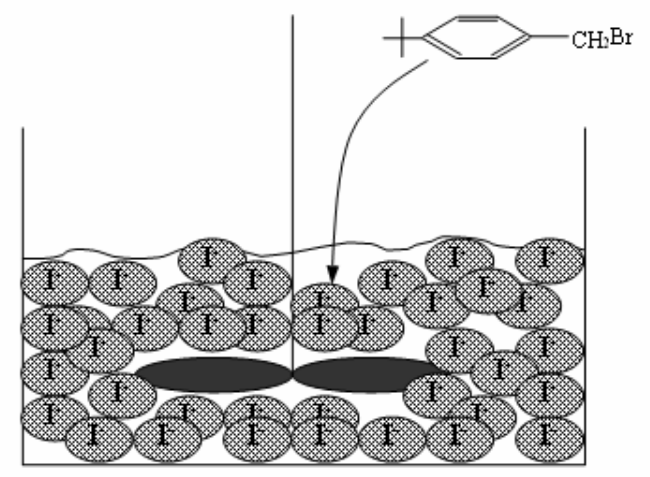

Figure 1. Reaction between 4-tert-butylbenzyl bromide and potassium iodide performed in a slurry of ordered mesoporous material.

Figure 2 shows the reaction profiles obtained in different types of reaction media. As can be seen the reaction is extremely rapid in a liquid crystalline system, no doubt due to the very large 
interface between the polar and the apolar domains of such systems. It is interesting to note that reaction in the slurry of mesoporous material is faster than that in the microemulsion. Microemulsions, which are thermodynamically stable mixtures of oil,water and surfactant, ${ }^{3}$ are today relatively established as medium for reactions that suffer from a reactant incompatibility problem. ${ }^{4-8}$
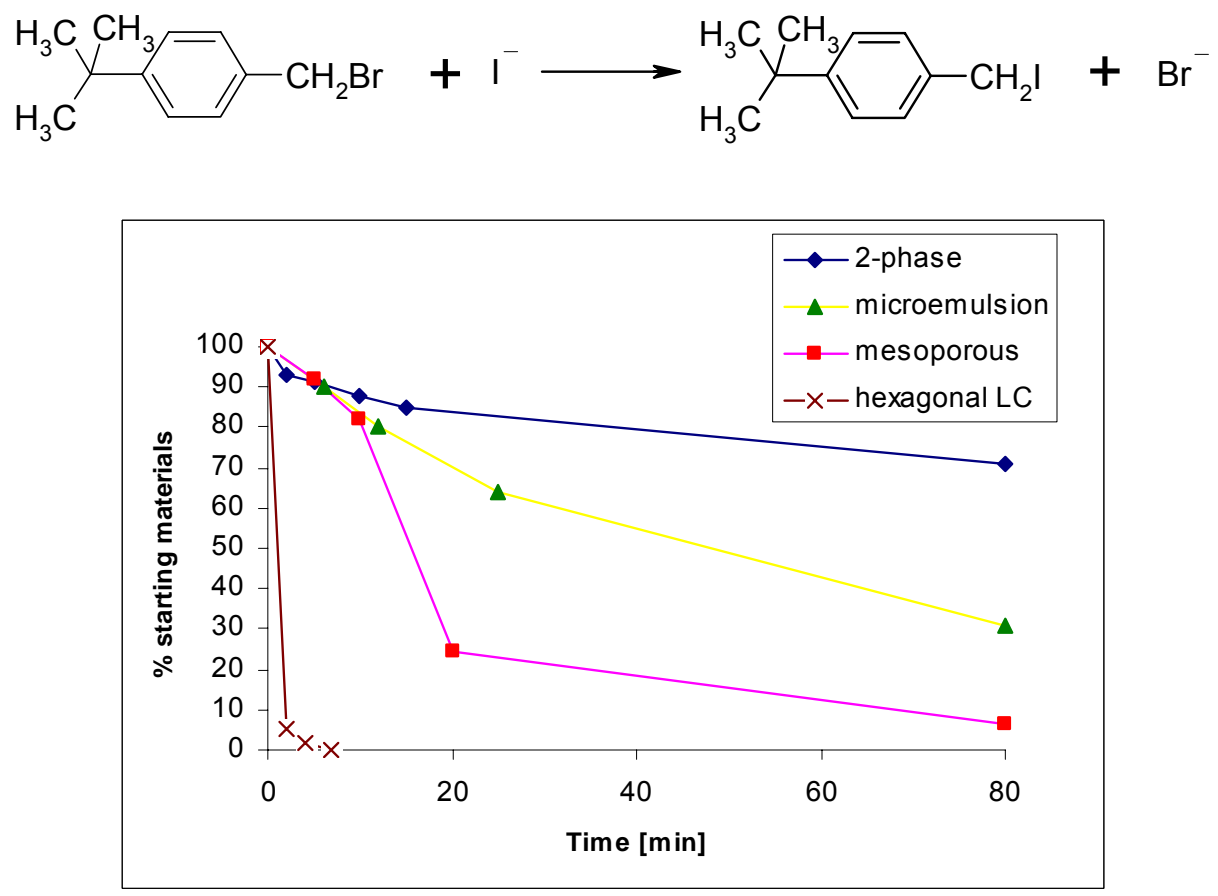

Figure 2. Profiles for the reaction between 4-tert-butylbenzyl bromide and potassium iodide performed in a two-phase system, a microemulsion, a slurry of mesoporous silica, and a hexagonal liquid crystalline phase. A block copolymer containing polyoxyethylene and polyoxypropylene segments was used as amphiphile for formation of the liquid crystal and penta(ethylene glycol)monododecyl ether was used as microemulsion surfactant. ${ }^{2}$

Figure 3, top shows that the geometry of the liquid crystalline phase or the mesoporous oxide material is not important for the reaction rate. One may have expected that the bicontinuous cubic structure, which is isotropic with pore openings in all three dimensions would be superior to the hexagonal structure, in which the pores are aligned in only one dimension. However, the geometry seems not to be decisive of the reaction rate. Figure 3, bottom shows the rates obtained with three different oxide materials. We have no good explanation for the low reactivity obtained with the slurries of mesoporous titania. The lower surface area of this material may contribute to the effect but most likely there are other factors involved also. One may expect that surface hydrophilicity could play a role but we were not able to detect any difference in wetting characteristics of the titania as compared to the silica or the alumina. We have also no explanation to why alumina, despite the considerably lower surface area, gives higher reactivity than silica. Reference 9 gives an account of the experimental procedure used. 

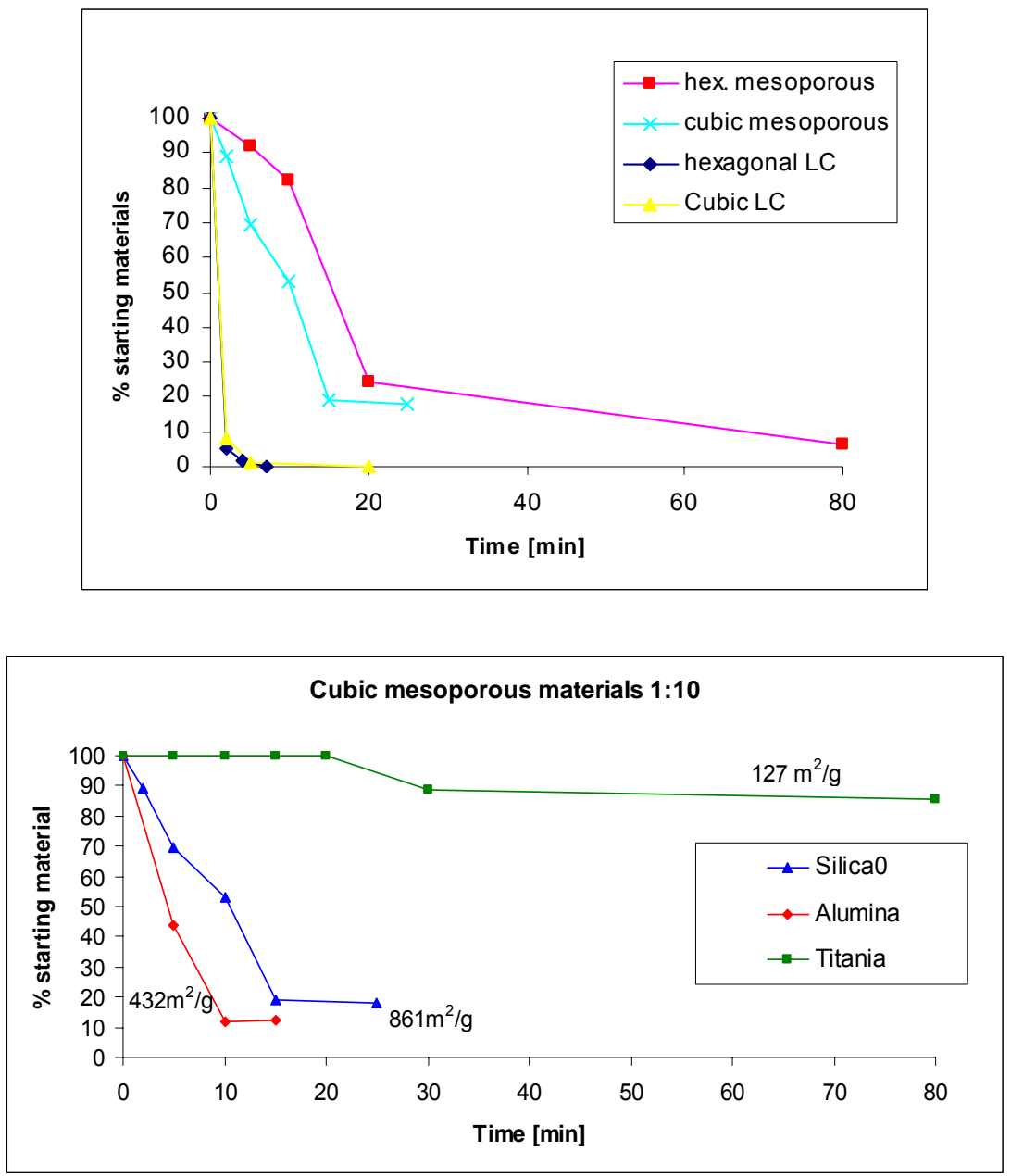

Figure 3. Top. Reaction profiles for the nucleophilic substitution reaction between 4-tertbutylbenzyl bromide and potassium iodide performed in slurries of hexagonal and cubic ordered mesoporous silica and hexagonal and cubic liquid crystalline phases. Bottom. The same reaction performed in slurries of ordered mesoporous silica, alumina, and titania with hexagonal symmetry.

The results displayed in Figures 2 and 3 show that a slurry of an ordered mesoporous oxide should be seen as alternative to the use of a two phase system with added phase transfer agent or to the use of a microemulsion as medium for reactions between incompatible reactants. The ease of workup - removal of the mesoporous material by filtration or centrifugation - is a clear advantage of the method. We have also demonstrated that the reaction can be performed by a column approach. ${ }^{2}$ The particles of KI-loaded mesoporous silica were packed in a column and 4-TBBB dissolved in hexane was passed through. The effluent contained almost pure product, 4-tertbutylbenzyl iodide.

Use of an ordered mesoporous oxide material as host for a metal-organic homogeneous catalyst

A Heck-type reaction comprising a carbon-carbon coupling between an arylboronic acid and styrene (Figure 4) was performed using a water-soluble rhodium catalyst entrapped in the water- 
filled pores of mesoporous silica particles. ${ }^{10}$ The catalyst used was $[\mathrm{Rh}(\mathrm{COD}) \mathrm{Cl}]_{2}$ with bis( $p$ sulfonatophenyl)phenylphosphine dipotassium salt (TPPDS) as water-solubilizing ligand. Thus, the water-filled silica pores can be seen as a host for the Rh catalyst, acting as a guest.

The catalyst-loaded inorganic particles were dispersed in a nonpolar medium, either a solution of the reactants in an aromatic solvent (toluene or $p$-xylene) or a solvent-free mixture of the reactants using a large excess of styrene.<smiles>COc1ccc(-c2ccc(C=Cc3ccc(C=Cc4cccc(OC)c4)cc3)cc2)cc1</smiles>

Figure 4. The Heck-type carbon-carbon coupling reaction of 4-methoxyphenylboronic acid and styrene yielding either the cis or trans isomer of the product 4-methoxystilbene. Also shown is the biphenylic biproduct.

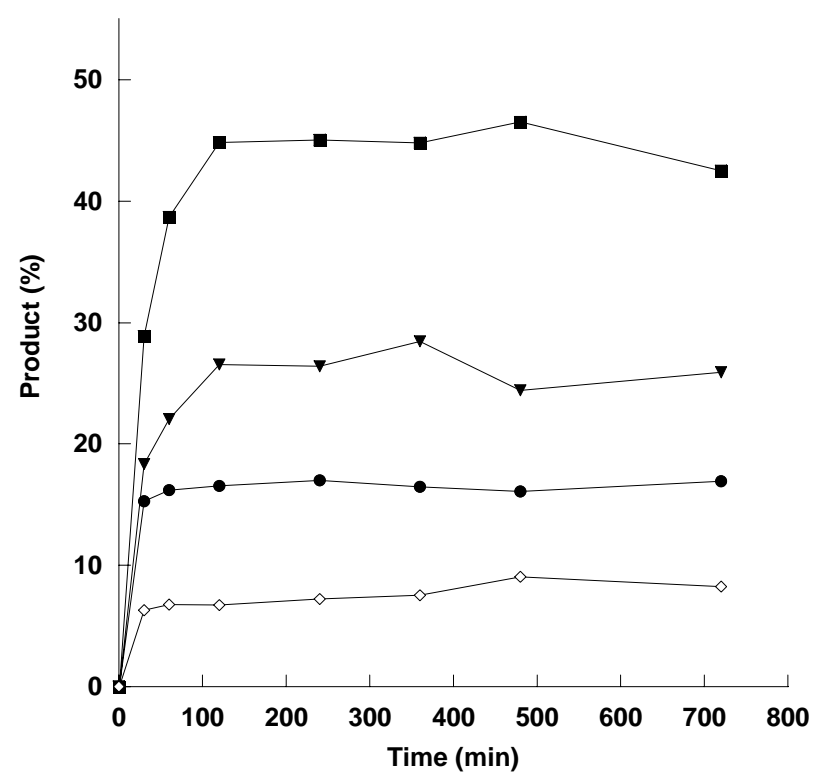

Figure 5. Reaction profile for the Rh catalyzed reaction between 4-methoxyphenylboronic acid and styrene in absence of solvent. $\mathbf{m}$ and $\mathbf{\nabla}$ show reactions with mesoporous silica using 50 and 25 eq. of styrene, respectively. $\bullet$ and $\diamond$ denote reactions employing a liquid-liquid two-phase system and non-porous silica, respectively, both using 25 eq. of styrene. Product (\%) refers to yield of 4methoxystilbene. All systems contained 2 mole $\%$ of the Rh catalyst. For the mesoporous and the non-porous silica systems catalysts loadings of $0.025 \mathrm{wt} \%$ were used.

From the results shown in Figure 5 it is obvious that the concept of using a mesoporous inorganic material as host for the Rh catalyst is a feasible approach. The pores were filled with an aqueous solution of the Rh catalyst, coordinated to charged ligands. The yield obtained was higher than that obtained in the conventional liquid-liquid two-phase system, see Figure 5. It is, however, 
likely that a thin water film will be present on the outside of the mesoporous silica particles and the ligand-bound $\mathrm{Rh}$ catalyst may then also partition into this film from where it can exert its action. A control experiment employing non-porous silica particles does indicate that the catalytic effect exerted by the $\mathrm{Rh}$ catalyst may partly be due to $\mathrm{Rh}$ present in the thin water film outside the particles. This contribution seems to be small, however. As shown in Figure 5, with non-porous silica 4-methoxystilbene was synthesized in a $7 \%$ yield after $2 \mathrm{~h}$, while with mesoporous silica a yield of $27 \%$ was obtained in the experiments with a 25:1 styrene:4-methoxyphenylboronic acid ratio. The particle size of the mesoporous material was approximately ten times larger than that of the non-porous material, which means that the latter had two orders of magnitude larger outer surface.

Taken together, this implies that for the reactions employing mesoporous silica the contribution from the Rh present in the water film outside the particles can be neglected. Previous reports have shown that the use of a water soluble Rh catalyst adsorbed on non-porous silica could generate high yields and good selectivity, as well as high reaction rates, for the hydroformylation of acrylate esters. ${ }^{11}$ The catalyst was believed to reside entirely in the thin water film surrounding the nonporous particles. However, for the carbon-carbon coupling reaction studied in this work, the porosity of the carrier material was obviously essential. We believe that the pore openings were the primary reaction zones.

\section{Use of a hydrophobized mesoporous material as host for a metal-organic homogeneous catalyst}

An 1,4-conjugate addition between two water soluble reactants, 4-carboxyphenylboronic acid and methyl acrylate, was performed using a homogeneous rhodium catalyst entrapped in the pores of hydrophobized ordered mesoporous silica, see Figure 6. The hydrophobation of the silica was made by a conventional silanization procedure. The catalyst-loaded particles were dispersed in an aqueous solution containing the reactants.

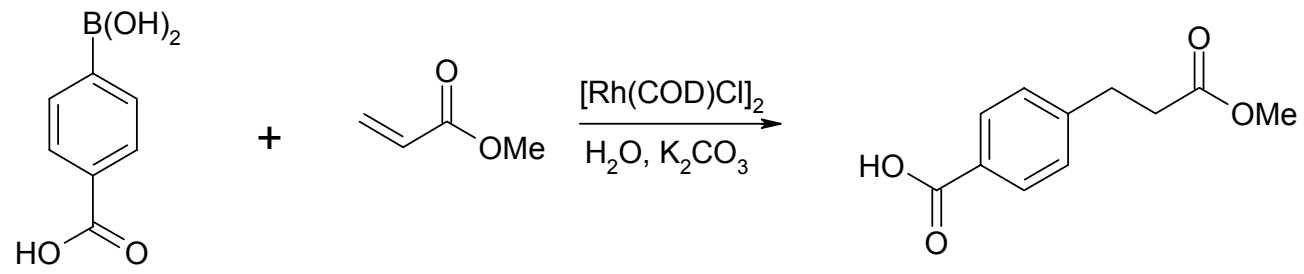

Figure 6. A rhodium catalysed coupling reaction between 4-carboxyphenylboronic acid and methyl acrylate.

The yields were compared with those obtained with a conventional liquid-liquid two-phase system. As can be seen from Figure 7, the system based on mesoporous material gave only marginally higher yield. The porous particles, containing the catalyst, are simply removed by filtration after completed reaction and the product is isolated as a precipitate obtained by acidification of the filtrate. As shown in Figure 7, the reusability is much better with the catalyst entrapped in the hydrophobized, mesoporous silica than with the conventional procedure where the catalyst is present in the apolar phase. 


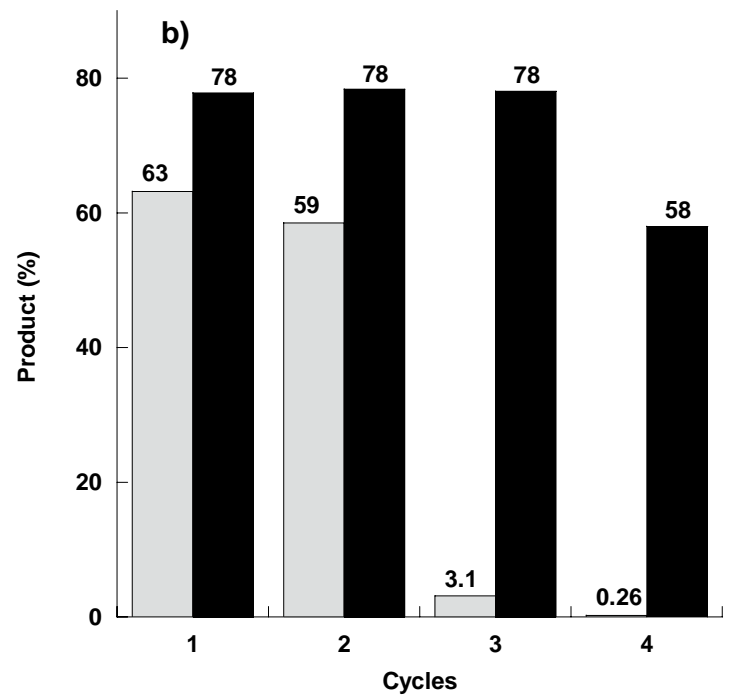

Figure 7. Yields obtained for four consecutive runs of the reaction between 4carboxyphenylboronic acid and methyl acrylate with the catalyst present either in a toluene phase, which is in equilibrium with an aqueous phase in which the reactants are present (grey bars), or in a toluene solution immersed in the pores of hydrophobized mesoporous silica and with the mesoporous particles dispersed in an aqueous phase in which the reactants are present (black bars).

\section{Use of palladium nanoparticles deposited inside the pores of ordered mesoporous carbon as a catalyst for the Sonogashira reaction}

A Sonogashira carbon-carbon coupling reaction between iodobenzene and phenylacetylene was performed using palladium nanoparticles formed in situ inside the pores of ordered mesoporous carbon as catalyst, see Figure 8.

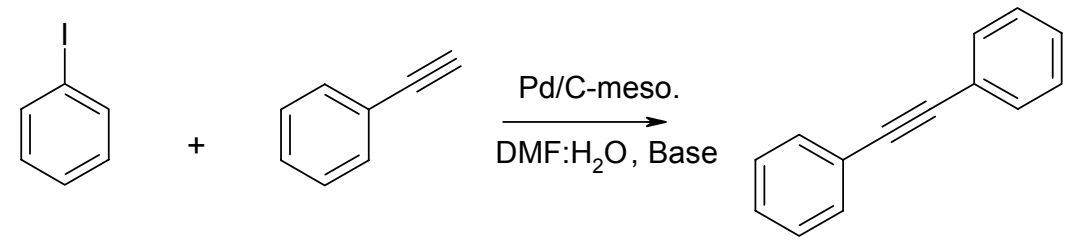

Figure 8. A Sonogashira reaction between iodobenzene and phenylacetylene catalyzed by palladium nanoparticles deposited inside the pores of mesoporous carbon.

The mesoporous carbon was formed by a double templating procedure. First a mesoporous silica was formed by the normal route using a nonionic block copolymer of polyoxyethylenepolyoxypropylene-polyoxyethylene type as structure directing agent. The pores were then impregnated with furfuryl amine, which was subsequently polymerized. The solid polymer was pyrolyzed to yield amorphous carbon. Polymerized furfuryl amine contains oxygen and nitrogen. Carbon dioxide and nitrogen-containing compounds will consequently be released during the pyrolysis, resulting in cavities in the amorphous carbon. In order to fill these cavities, the material was again impregnated with furfuryl amine followed by polymerization and pyrolysis. The 
procedure was repeated three times, resulting in a well-defined carbon-silica composite material. The silica was removed by treatment with hydrofluoric acid, resulting in a fine black powder of ordered mesoporous carbon with a specific surface area of approximately $980 \mathrm{~m}^{2} / \mathrm{g}$ and a pore diameter around $3 \mathrm{~nm}$.

The mesoporous carbon was impregnated with a water-in-oil microemulsion containing $\mathrm{PdCl}_{2}$ in the water pools. A second water-in-oil microemulsion was added that contained $\mathrm{NaBH}_{4}$ in the small water droplets. This procedure is an example of the microemulsion route of synthesizing nanoparticles, ${ }^{12}$ and the $\operatorname{Pd}(0)$ particles formed became deposited on the pore walls, as seen by electron tomography. ${ }^{13}$

The Sonogashira reaction using the Pd-loaded mesoporous carbon as catalyst and using $\mathrm{NaOAc}$ as base gave a yield of $48 \%$. An experiment with Pd nanoparticles suspended in the reaction mixture gave $28 \%$ yield. The moderate yields in both reactions were mainly due to incomplete conversion of the starting material, not to formation of by-products. Most likely, the discontinuation of the reaction was caused by catalyst inactivation. It is known that under the conditions of the Sonogashira reaction the catalyst may be deactivated by formation of large polydisperse crystalline Pd particles with small surface area, often referred to as palladium black. ${ }^{14}$ The reduced catalytic activity of palladium black may arise from the crystalline particles having a low degree of active sites, such as steps, planes and edges.

It has been suggested that for coupling reactions using nanoparticles, the truly active catalyst may be species leached from the solid particles. ${ }^{15-17}$ One facile and useful method to prove the presence of leached and catalytically active species is the so-called Maitlis' filtration test. ${ }^{18}$ The test is based on adding new reactants to a newly filtered reaction solution and subsequently monitoring formation of the reaction product. A fresh set of reactants, but no catalyst, was added to the filtrate and the reaction was allowed to continue for $17 \mathrm{~h}$. A $50 \%$ additional yield was obtained with $\mathrm{NaOAc}$ as base. These results show that the Sonogashira reaction studied in this work is based on homogeneous catalysis, or, to be more cautious, that homogeneous catalysis is at least a strongly contributing reaction pathway. The degree of leaching was quantified by SEM-EDX.

\section{Biocatalysis with a lipase entrapped in the pores of mesoporous oxide materials}

Three mesoporous materials; silica, alumina, and titania, were used as host for a lipase from Rhizomucor miehei and the enzyme-loaded particles were employed as catalyst for esterification of caprylic acid using a mixture of glycerol and water as reaction medium. ${ }^{19}$ The reaction proceeded well with all three oxides but alumina gave considerably higher conversion than the other two, see Figure 9. Hydrophobized silica gave an even higher degree of esterification. The degree of esterification obtained is believed to depend on the microenvironment of the enzyme. When alumina, which is positively charged under the conditions used, and hydrophobized silica are used as host material, the negatively charged lipase can be assumed to be adsorbed at the walls of the pores. The water activity is believed to be lower at the solid surface than in the middle of the pores, where the enzyme is situated when silica and alumina are used as host material. 


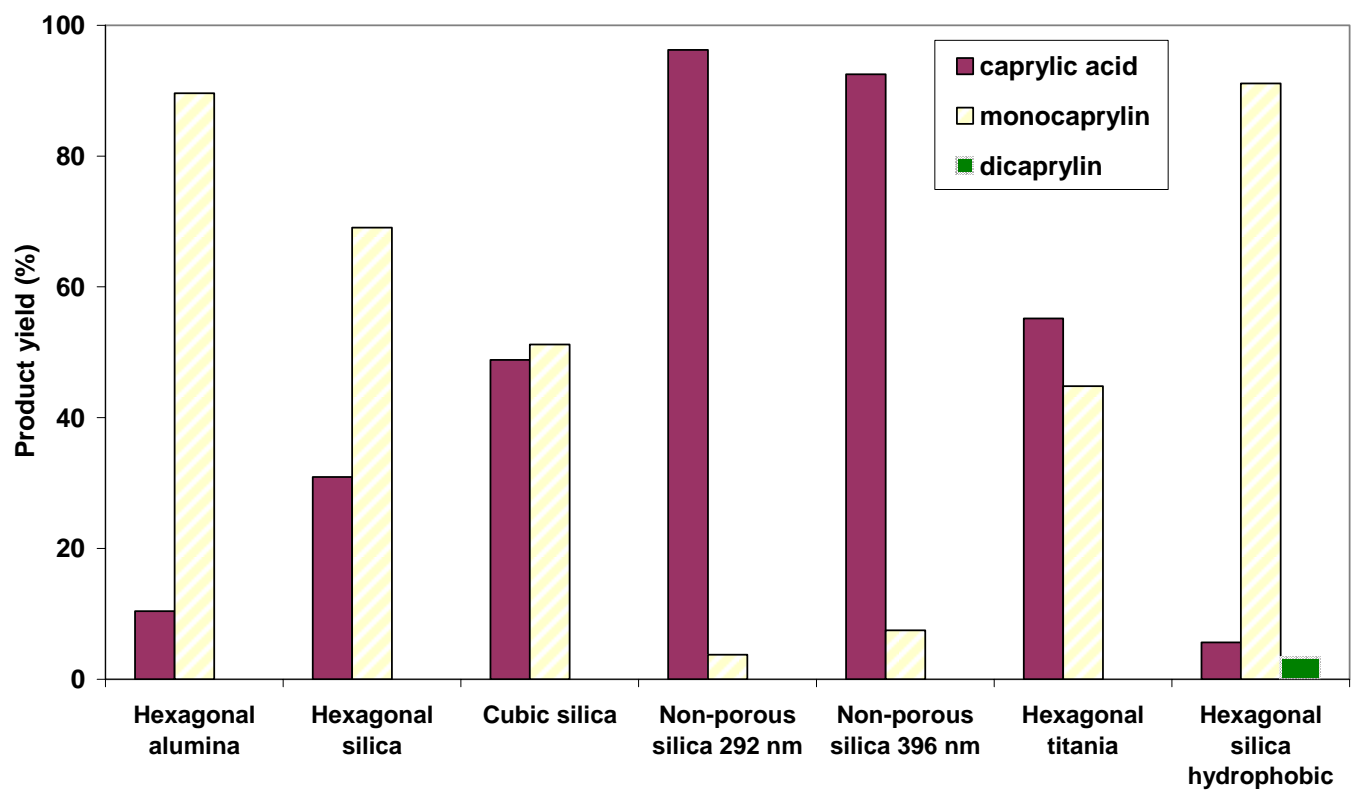

Figure 9. Effect of the type of mesoporous material on the relative amounts of caprylic acid and monocaprylin formed when caprylic acid was used as substrate and a water/glycerol ratio of 15:85, corresponding to an $\mathrm{a}_{\mathrm{w}}$ of 0.36 , was used as reaction medium. Reactions with nonporous silicas were performed as references.

It was found that the lipase was not irreversibly entrapped in the pores of the mesoporous materials. When the particles were removed by filtration after completed reaction and subsequently washed with an aqueous buffer, the enzyme leached out. The lipase could be immobilized in the pores, however, by cross-linking in-situ inside the pores using glutaraldehyde as cross-linking agent, using a procedure taken from the literature. ${ }^{20}$ Glutaraldehyde links proteins together via formation of Schiff bases involving primary amino groups. Figure 10 is a schematic of cross-linked lipase situated inside the pores of mesoporous material with hexagonal geometry.

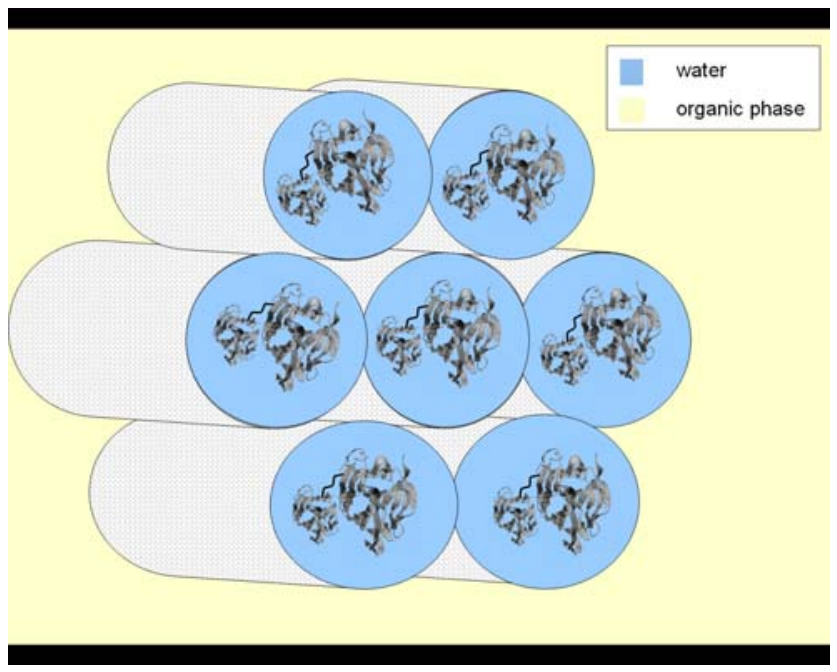

Figure 10. Cartoon showing cross-linked lipase inside the pores of hexagonal mesoporous material. 
The cross-linked lipase inserted into the mesoporous material showed excellent reusability. Hydrolysis of 4-nitrophenylpalmitate was used as test reaction since the 4-nitrophenol generated gives the reaction medium a strong yellow colour, easy to monitor spectroscopically. As can be seen from Figure 11, the activity stayed at almost the same level during five consecutive runs. The results with the lipase present in the hexagonal alumina were particularly consistent. The crosslinking is evidently a way to prevent the enzyme from escaping the pores without impairing its catalytic activity. Thus, it seems that particles of ordered mesoporous oxide materials loaded with cross-linked lipase are well suited for biotransformation with repeated use of the catalyst.

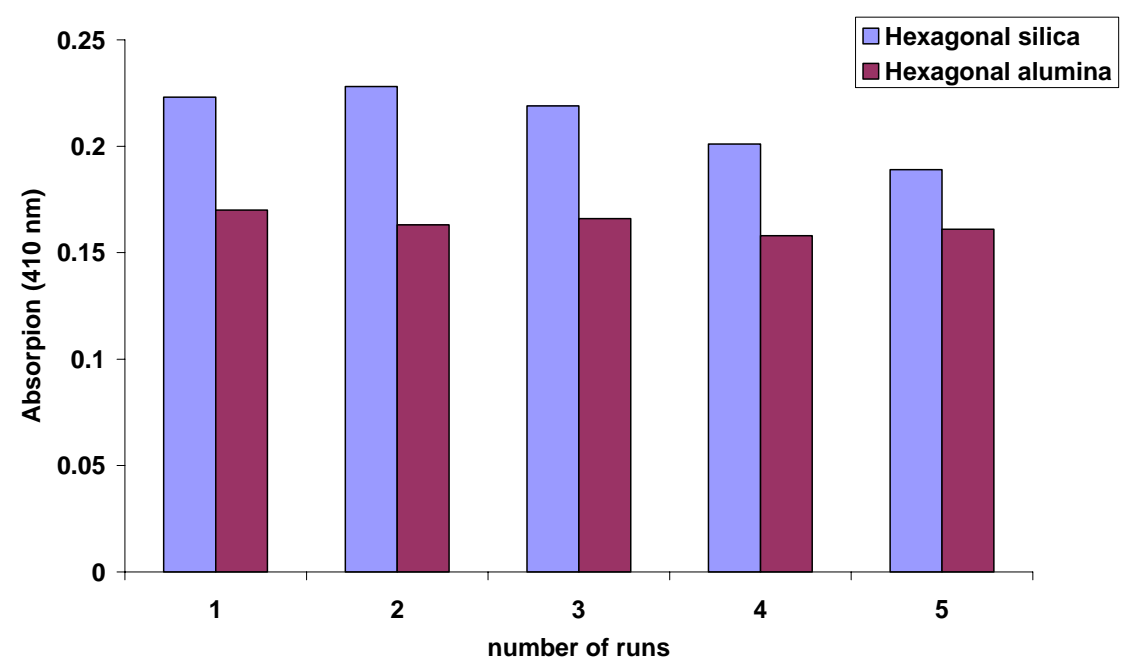

Figure 11. Relative amounts of 4-nitrophenol generated by lipase catalyzed hydrolysis of 4nitrophenylpalmitate. The enzyme was embedded in either hexagonal silica or hexagonal alumina and subsequently cross-linked. The enzyme-loaded particles were separated by filtration and washed with aqueous buffer between each run.

\section{Conclusions}

We have shown in a series of recent papers and in on-going work that ordered mesoporous materials can be useful tools for organic synthesis. Their main advantage lies in the ease of work-up and the potential for reuse. A homogeneous catalyst, such as a synthetic metal-organic compound or an enzyme can be heterogenized inside the pores and the catalyst will exert its action at the pore openings. This requires that the reactants and the products are soluble in the continuous phase and that they do not partition into the phase that fills the pores. The principle is illustrated in Figure 12. Since the pores can be either hydrophilic or hydrophobic they can be host for both polar and apolar catalysts, as has been demonstrated in this paper. 


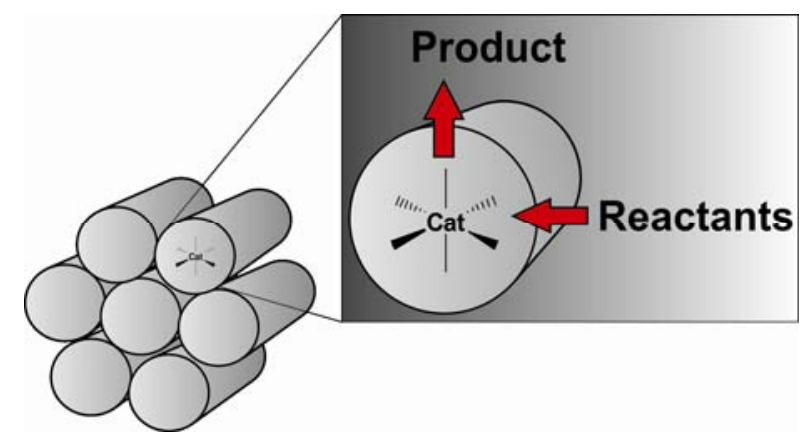

Figure 12. A homogeneous catalyst inserted into the pores of an ordered mesoporous material with hexagonal geometry. Both the reactant and the product partition into the phase that surrounds the particles of mesoporous material and the reaction occurs at the pore openings.

For work in laboratory scale the catalyst-loaded particles are conveniently removed by filtration or centrifugation, washed with the solvent that constitutes the continuous phase of the slurry and reused. For larger scale work a column procedure might be a more attractive option. ${ }^{2}$

\section{Acknowledgements}

We would like to thank the Swedish Research Council, the Swedish Foundation for Strategic Research and Nestlé S.A. for support to PH, TW and PR, respectively.

\section{References}

1. Soler-Illia, G. J. A. A.; Sanchez, C.; Lebeau, B. Chem. Rev. 2002, 102, 4093.

2. Witula, T.; Holmberg, K. Langmuir 2005, 21, 3782.

3. Danielsson, I.; Lindman, B. Colloids Surfaces 1981, 3, 391

4. Ruasse, M. F.; Blagoeva, I. B.; Ciri, R.; Garcia-Rio, L.; Leis, J. R.; Marques, A.; Mejuto, J.; Monnier, E. Pure Appl. Chem. 1997, 69, 1923.

5. Bode, G.; Lade, M.; Schomäcker, R. Chem. Eng. Technol. 2000, 23, 405.

6. Holmberg, K. Curr. Opin. Colloid Interface Sci. 2003, 8, 187.

7. Dwars, T.; Paetzold, E.; Oehme, G. Angew. Chem. Int. Ed. 2005, 44, 7174.

8. Holmberg, K. Eur. J. Org. Chem. 2007, 731.

9. Witula, T.; Holmberg, K. Langmuir 2007, 310, 536.

10. Handa, P.; Stjerndahl, M.; Holmberg, K. Microporous Mesoporous Mater. 2007, 100, 146.

11. Fremy, G.; Monflier, E.; Carpentier, J.-F.; Castanet, Y.; Mortreux, A. Angew. Chem. Int. Ed. 1995, 34, 1474.

12. Pileni, M.-P. J. Phys. Chem. 1993, 97, 6961.

13. Wikander, K.; Hungria, A. B.; Midgley, P. A.; Palmqvist, A. E. C.; Holmberg, K.; Thomas J. M. J. Colloid Interface Sci. 2007, 305, 204.

14. Beletskaya, I. P.; Cheprakov, A. V. Chem. Rev. 2000, 100, 3009. 
15. Thathagar, M. B.; Kooyman, P. J.; Boerleider, R.; Jansen, E.; Elsevier, C. J.; Rothenberg, G. Adv. Synth. Catal. 2005, 347, 1965.

16. Thathagar, M. B.; ten Elshof, J. E.; Rothenberg, G. Angew. Chem. Int. Ed. 2006, 45, 2886.

17. Gaikwad, A. V.; Holuigue, A.; Thathagar, M. B.; ten Elshof, J. E.; Rothenberg, G. Chem. Eur. J. 2007, 13, 6908.

18. Hamlin, J. E.; Hirai, K.; Millan, A.; Maitlis, P. M. J. Mol. Catal. 1980, 7, 543.

19. Reis, P.; Witula, T.; Holmberg, K. Micropor. Mesopor. Mater. 2007, 110, 355.

20. Lopez-Serrano, P.; Cao, L.; van Rantwijk, F.; Sheldon, R. A. Biotechnol. Lett. 2002, 24, 1379. 